\title{
Intervenção fisioterápica e prevenção de quedas em idosos
}

\author{
Physical therapy intervention and elderly falls prevention
}

\author{
Intervención fisioterápica y prevención de caídas en adultos mayores
}

\author{
Samária Ali Cader*, Jorge da Silva Barboza, Adalgisa leda M. Bromerchenkel
}

\begin{abstract}
Resumo
As quedas são um problema de saúde pública entre os idosos, em vista da mortalidade, morbidade e dos custos social e econômico. O tema é muito valorizado pela gerontologia e uma fonte de preocupação para os pesquisadores dessa área, principalmente quando pessoas denominam esse evento como sendo normal e próprio do processo de envelhecimento. Queda pode ser definida como um evento não intencional que tem como resultado a mudança de posição do indivíduo para um nível mais baixo em relação a sua posição inicial. Pessoas de todas as idades apresentam risco de sofrer queda. Porém, para os idosos, elas possuem um significado muito relevante, pois podem levá-los à incapacidade, injúria e morte. Seu custo social é imenso e torna-se maior quando o idoso tem diminuição da autonomia e da independência ou passa a necessitar de institucionalização. Sabe-se que é elevado o número de idosos que caem e que mudam radicalmente sua vida cotidiana, tanto pela queda em si como pelo temor de uma nova ocorrência, restringindo suas atividades, aumentando o isolamento social e o declínio na saúde, repercutindo no risco de serem institucionalizados. Dentre os fatores de risco, aqueles de maior ocorrência são a deterioração da visão, uso simultâneo de medicamentos (especialmente diuréticos e psicoativos) e flexibilidade reduzida (quadril e tornozelos), fatores estes que deveriam ser considerados em programas para prevenção de quedas em idosos. Além de prejuízo físico e psicológico, esses acidentes geram um aumento dos custos com cuidados de saúde, expressos pela utilização de serviços especializados e aumento de hospitalizações. Assim, são numerosos os estudos que analisam as quedas em idosos, tanto no que diz respeito à epidemiologia, etiologia e fatores de risco associados, como em relação às consequências desses eventos. O presente estudo teve como objetivo fazer uma revisão sobre o histórico de quedas de idosos e sugerir de que forma a fisioterapia interferirá para minimizar a ocorrência das mesmas.
\end{abstract}

Descritores: Envelhecimento; Autonomia pessoal; Saúde pública; Acidentes por quedas; Fisioterapia; Fatores de risco.

\begin{abstract}
Falls are a public health problem among the elderly, in view of mortality, morbidity and social and economic costs. The theme is highly valued by gerontology and a source of concern for researchers in this area, especially when people consider this event normal and part of the aging process. A fall can be defined as "one unintentional event which results in the change in position of the individual to a lower level in relation to its initial position." People of all ages are at risk of falling. However, for the elderly, it has a much greater relevance as they may lead to disability, injury and death. The social cost is enormous and becomes larger when the elderly have decreased autonomy and independence or need institutionalization. It is known that a high number of elderly people fall and radically change their daily lives, both for the fall itself and for the fear of a recurrence, restricting their activities and increasing social isolation and health deterioration, impacting the risk of being institutionalized. Among the risk factors, the most frequent are deterioration of vision, simultaneous use of drugs (especially diuretics and psychoactive), and reduced flexibility (hip and ankle), factors that should be considered in programs to prevent falls in elderly populations. Besides physical and psychological damage, these accidents generate higher costs for health care, expressed by the use of specialized services and an increase in hospitalizations. Thus, there are several studies analyzing falls in the elderly, both with regard to epidemiology, etiology and risk factors and the consequences of these events. This study explores the process of investigation of falls in the elderly and aims to review the history of falls in the elderly population and suggest how physiotherapy will intervene to minimize their occurrence.
\end{abstract}


Keywords: Aging; Personal autonomy; Public health; Accidental falls, Physical therapy specialty; Risk factors.

\section{Resumen}

Las caídas son un problema de salud pública entre los adultos mayores, en vista de la mortalidad, morbidad y de los costos sociales y económicos. El tema es muy apreciado por la gerontología y una fuente de preocupación para los investigadores de esta área, principalmente cuando las personas denominan ese suceso como normal y propio del proceso de envejecimiento. Una caída puede ser definida como un suceso involuntario que resulta del cambio de posición de la persona a un nivel más bajo, en relación con su posición inicial. Personas de todas las edades corren el riesgo de sufrir caídas. Sin embargo, para los adultos mayores éstas poseen un significado muy relevante, pues pueden llevarlo a la incapacidad, injuria y muerte. Su costo social es enorme y se vuelve mayor cuando en el adulto mayor han disminuido su autonomía e independencia o requiere de institucionalización. Se sabe que es elevado el número de adultos mayores que caen y cambian radicalmente su vida cotidiana, tanto por la caída en si, como por el temor a un nuevo incidente, restringiendo sus actividades, aumentando el aislamiento social y el declive en la salud, repercutiendo en el riesgo de ser institucionalizados. Entre los factores de riesgo, los de mayor ocurrencia son la deterioración de la visión, el uso simultáneo de medicamentos (especialmente diuréticos y psicoactivos) y la flexibilidad reducida (cuadril y tobillos), factores que deberían ser considerados en los programas para la prevención de caídas en adultos mayores. Además del daño físico y psicológico, esos accidentes generan un aumento de los costos en asistencia sanitaria, reflejados en la utilización de servicios especializados y el aumento de hospitalizaciones. Así, los estudios que analizan las caídas en adultos mayores son numerosos, tanto en lo que se refiere a la epidemiología, etiología y factores de riesgo asociados, como con relación a las consecuencias de estos sucesos. El presente estudio tuvo como objetivo realizar una revisión sobre el historial de caídas en adultos mayores y sugerir en qué forma la fisioterapia interfiere para minimizar la ocurrencia de las mismas.

Palabras clave: Envejecimiento; Autonomía personal, Salud pública; Accidentes por caídas; Fisioterapia; Factores de riesgo.

\section{Introdução}

A queda em idosos é uma ocorrência comum, especialmente em hospitais e em casas de repouso, ${ }^{1}$ sendo ainda uma causa frequente de admissão nestas instituições. ${ }^{2}$ Os fatores causadores da queda podem ser de ordem fisiológica e/ou biológica ou psicológica e/ou social, e incluem: diminuição de força muscular; desequilíbrio; uso de bengala, andadores e muletas; deficiência visual e cognitiva; medo de cair; depressão; comportamento sedentário; idade; número de medicamentos (psicotrópicos, para comprometimentos cardiovasculares); deficiência nutricional; incontinência urinária; artrite e tipo de calçado.,

O processo natural do envelhecimento, associado à inatividade física, pode, gradualmente, levar à redução do desempenho físico, uma forte causa para o episódio de queda, ${ }^{5}$ sendo esta incidência maior após os 75 anos. ${ }^{6}$ Dentre as principais consequências da queda, pode-se destacar: as fraturas, o hematoma subdural, a cegueira, o comprometimento funcional, a ansiedade, a depressão, o aumento da permanência hospitalar e a inabilidade para retornar à residência (aumentando os custos com saúde). ${ }^{7}$
Dentre os instrumentos de avaliação do risco de quedas, o Timed Up And Go Test (TUGT) é o único teste recomendado pela American Geriatrics Society e pela British Geriatrics Society para avaliação do risco de quedas incluindo itens funcionais com alta relação ao número de quedas como um componente de marcha importante, ${ }^{8}$ devido à ocorrência de quedas durante a deambulação e dupla tarefa associada a situações diárias envolvendo demandas simultâneas. ${ }^{9}$ Ao contrário da Berg Balance Scale (BBS), não apresenta efeito teto e é um teste objetivo devido à cronometragem do tempo para execução da tarefa, o que o torna mais sensível como instrumento. ${ }^{10} \mathrm{O}$ TUGT consome menor tempo de aplicação, quando comparado à BBS, que leva de 15 a 20 minutos. ${ }^{11}$ Um aspecto limitante da BBS é a falta de um teste que avalie a execução de múltiplas tarefas. ${ }^{2}$ Diferentemente de outros testes, como o TUGT, a BBS faz uso da observação do avaliador para a análise do desempenho da atividade. ${ }^{10}$

A prática da atividade física na terceira idade pode levar ao bem-estar físico e mental e à autoconfiança por meio do domínio do corpo, aumento de prontidão para atividades da vida diária (AVD), 
maior disposição, maior mobilidade articular, intensificação da circulação sanguínea (sobretudo nas extremidades), aumento das capacidades de coordenação e reação, e por meio do combate a depressão, medo, decepções, aborrecimentos, tédio e solidão, pois, além de tudo, leva o geronte à reintegração social na comunidade. ${ }^{13}$ Por conseguinte, as alterações proporcionadas pelo treinamento funcional no controle neuromotor são refletidas na melhora das capacidades funcionais e na diminuição da propensão a quedas, repercutindo na melhora da qualidade de vida dos idosos. ${ }^{14}$

Existem poucos estudos destinados à investigação dos principais fatores que levam à queda em idosos não hospitalizados ${ }^{15} \mathrm{e}$ da abordagem da fisioterapia na prevenção de quedas em idosos. Neste sentido, a proposta do presente estudo foi analisar desde o histórico de quedas até suas profilaxias por meio de acompanhamento fisioterapêutico e orientações.

\section{Fatores de risco e causas para a queda em idosos}

Apesar do interesse no assunto, nota-se certa divergência nas abordagens utilizadas para investigar os fatores de risco para as quedas. As publicações divergem, por exemplo, quanto aos critérios metodológicos utilizados, principalmente em relação à seleção da amostra: enquanto algumas se voltam para idosos vivendo em asilos, hospitais e casas de repouso, ${ }^{16,17}$ outras observam sujeitos vivendo na comunidade..$^{18}$ Além disso, as quedas ocorrem, ao menos em parte, em função de limitações fisiológicas de equilíbrio, força, visão ou tempo de reação, bem como em decorrência de doenças e, em certos casos, das estratégias terapêuticas para lidar com elas. Fatores como idade, sexo, doenças, uso de medicamentos, visão deficiente, prejuízo da capacidade funcional, pouco equilíbrio, fraqueza muscular e perigos ambientais têm sido apontados pela literatura como predisponentes de quedas em maior grau. Isso pode variar de grupo para grupo, sendo importante conhecer as peculiaridades dos idosos com os quais se lida a fim de se definirem estratégias que possam contribuir para a diminuição do risco..$^{19}$

Uma série de estudos tem identificado fatores de risco para quedas. Estes podem ser classificados como intrínsecos (por exemplo, fraqueza das extremidades inferiores, força de preensão fraca, distúrbios do equilíbrio, comprometimento funcional e cognitivo, deficit visual) ou extrínsecos (por exemplo, polifarmácia, ou seja, quatro ou mais medicamentos de prescrição) e ambientais (tais como má iluminação, tapetes soltos e falta de equipamentos de segurança para banheiro). Embora os investigadores não tenham usado de forma consistente, uma revisão de estudos de fatores de risco para queda classificou estes fatores conforme o quadro $1 .{ }^{20}$

No quadro $2,{ }^{20}$ estão sumarizadas as causas mais comuns de quedas em idosos e as drogas que mais provavelmente aumentam o seu risco.

\section{Estudos originais na abordagem da queda em idosos}

Semelhantemente aos objetivos da presente pesquisa, em um estudo da American Geriatrics Society foram sumarizadas as incidências dos principais fatores de risco para a queda (Tabela 1). ${ }^{4}$

Em um estudo realizado por Hitcho e colaboradores foi descrita a epidemiologia de quedas em pacientes hospitalizados incluindo as características dos pacientes que caem, as circunstâncias da queda e os comprometimentos relativos à mesma. ${ }^{21}$ Os dados foram coletados através de entrevistas com os pacientes, enfermeiros e relatos médicos. Durante o período de estudo, houve um total de 183 pacientes com história de queda, com idade média de 63,4 anos (17 a 96 anos). Os autores observaram que muitas quedas não foram assistidas (79\%) e ocorreram no quarto dos pacientes (85\%), durante a noite (59\%), e durante a deambulação (19\%). Metade das quedas (50\%) era mais comum em pacientes acima de 65 anos.

Perracini e Ramos, ${ }^{22}$ em um estudo que analisou os fatores relacionados a quedas em idosos residentes em comunidade, destacaram o comprometimento visual como um dos principais fatores de risco. De acordo com seus resultados, idosos com diminuição leve, moderada e severa na acuidade visual apresentaram, respectivamente, 1,4, 1,0 e 2,2 vezes mais chance de caírem duas ou mais vezes.

Laessoe e colaboradores ${ }^{23}$ utilizaram uma 


\section{Quadro 1. Fatores de risco para queda em idosos}

\section{Fatores de risco em idosos}

\section{Fatores demográficos}

Idade mais avançada (especialmente $\geq 75$ anos)

Raça branca

Viver sozinho

\section{Fatores históricos}

Uso de bengala ou andador

Quedas anteriores

Doença aguda

Condições crônicas, distúrbios (especialmente neuromusculares)

Medicamentos, especialmente o uso de quatro ou mais medicamentos

\section{Déficit físicos}

Comprometimento cognitivo

Visão reduzida, incluindo mudanças relacionadas à idade (ou seja: diminuição da acuidade visual, diminuição da capacidade de acomodação, intolerância ao glare, percepção de profundidade alterada, presbiopia [visão de perto], diminuição da visão noturna, diminuição da visão periférica)

Dificuldade de se levantar de uma cadeira

Problemas nos pés

Alterações neurológicas, incluindo mudanças relacionadas à idade (ou seja: instabilidade postural, tempo de reação retardado, percepção sensorial diminuída para toque de luz, vibração e temperatura, declínio da central de integração visual e dos sentidos vestibular e proprioceptivo)

Diminuição da audição, incluindo mudanças relacionadas à idade (ou seja, a presbiacusia [aumento no limiar de tom puro, frequência predominantemente alta], discriminação de fala comprometida, acumulação excessiva de cerume)

\section{Outros}

Riscos ambientais

Comportamentos de risco

Fonte: adaptado de Fuller ${ }^{20}$

\section{Quadro 2. Causas mais comuns de quedas em idosos e drogas que aumentam o risco de sua ocorrência}

\begin{tabular}{|c|c|}
\hline Causas comuns de quedas em idosos & Drogas que podem aumentar o risco de queda \\
\hline $\begin{array}{l}\text { Acidente, risco ambiental, queda da cama } \\
\text { Alterações na marcha, distúrbios do equilíbrio ou fraqueza, dor } \\
\text { associada à artrite } \\
\text { Vertigem } \\
\text { Medicamentos ou álcool } \\
\text { Doença aguda } \\
\text { Confusão e comprometimento cognitivo } \\
\text { Hipotensão postural } \\
\text { Desordem visual } \\
\text { Distúrbio do sistema nervoso central, síncope, ataques de gota, } \\
\text { epilepsia }\end{array}$ & $\begin{array}{l}\text { Sedativos hipnóticos e ansiolíticos (principalmente benzodiazepínicos de ação } \\
\text { prolongada) } \\
\text { Antidepressivos tricíclicos } \\
\text { Tranquilizantes maiores (fenotiazinas e butirofenonas) } \\
\text { Drogas anti-hipertensivas } \\
\text { Medicamentos cardíacos } \\
\text { Corticosteroides } \\
\text { Anti-inflamatórios não esteroides } \\
\text { Anticolinérgicos } \\
\text { Hipoglicemiantes } \\
\text { Qualquer medicamento capaz de afetar o equilíbrio }\end{array}$ \\
\hline
\end{tabular}

Fonte: adaptado de Fuller ${ }^{20}$ 
Tabela 1. Resultados da análise multivariada dos fatores de risco mais comuns

\begin{tabular}{|c|c|c|c|}
\hline Fator de risco & Significativo/total * & Média $\mathrm{RR} / \mathrm{OR} * *$ & Intervalo \\
\hline Fraqueza muscular & $10 / 11$ & 4,4 & $1,5-10,3$ \\
\hline História de quedas & $12 / 13$ & 3 & $1,7-7,0$ \\
\hline Déficit na marcha & $10 / 12$ & 2,9 & $1,3-5,6$ \\
\hline Déficit no equilíbrio & $8 / 11$ & 2,9 & $1,6-5,4$ \\
\hline Uso de auxílio para a marcha & $8 / 8$ & 2,6 & $1,2-4,6$ \\
\hline Déficit visual & $6 / 12$ & 2,5 & $1,6-3,5$ \\
\hline Artrite & $3 / 7$ & 2,4 & $1,9-2,9$ \\
\hline AVD*** comprometidas & $8 / 9$ & 2,3 & $1,5-3,1$ \\
\hline Depressão & $3 / 6$ & 2,2 & $1,7-2,5$ \\
\hline Cognição comprometida & $4 / 11$ & 1,8 & $1,0-2,3$ \\
\hline idade $>80$ anos & $5 / 8$ & 1,7 & $1,1-2,5$ \\
\hline
\end{tabular}

Fonte: adaptado da American Geriatrics Society ${ }^{21}$

* Número de estudos com significativo odds ratio (OR) ou risco relativo (RR) / número total de estudos que incluem cada fator ** RR/OR: risco relativo (estudos longitudinais) / odds ratio (estudos transversais)

*** AVD: atividades da vida diária

bateria de testes, com parâmetros fisiológicos, relacionados à queda e ao equilíbrio, para avaliar o risco de queda de uma população de idosos em uma comunidade. Durante um ano, foi avaliado um total de 94 idosos, homens e mulheres (idade entre 70 e 80 anos) e foi encontrada uma incidência de $15 \%$ de quedas. Entretanto, em seus resultados, foi observado que o equilíbrio, isoladamente, não constitui um forte fator de risco para queda, sendo compreendido como um fenômeno complexo de origem multifatorial. Na verdade, a circunstância crucial é que a incidência de quedas está relacionada com a correlação entre a capacidade de equilíbrio e a demanda de equilíbrio exigida, sendo dependente do estilo de vida e comportamento individual.

Stalenhoef e colaboradores ${ }^{24}$ propuseram a construção de um modelo de risco para quedas em idosos, em que os autores citam a fraqueza de músculos e de articulações como um fator de risco para quedas recorrentes em indivíduos com 70 anos ou mais. Semelhantemente, Thrane e colaboradores ${ }^{25}$ observaram uma associação significativa do teste Time Up And Go (TUG), que está relacionado à habilidade e mobilização, com a história de quedas apenas em homens, tendo uma relevância clínica desta associação muito limitada.

Ziere e colaboradores ${ }^{26}$ tiveram por objetivo avaliar a correlação do uso de múltiplas drogas (quatro ou mais por dia) com a prevalência de quedas. Em um estudo de coorte, foram entrevistados 697 idosos, com idade igual ou superior a 55 anos. Em seus resultados foi observado um risco de queda maior nas mulheres. A incapacidade, a presença de comprometimento articular, a história de fratura e o uso de dispositivos de marcha representaram fortes fatores de risco. O risco de queda também teve um aumento significativo com o uso de medicamentos ( $p<0,0001)$. Contudo, fato interessante é que esta correlação existia apenas quando ao menos um destes medicamentos favorecia o risco de quedas (diuréticos, por exemplo). Todavia, Fabrício e colaboradores ${ }^{7}$ vêm contrapor os resultados da presente pesquisa, pois verificaram, em sua amostra de 50 idosos de um hospital público, que o uso de medicamentos não possuía uma relação direta com o risco de quedas.

Em uma investigação com um grupo inicial de 72 sujeitos, Guimarães e Farinattii ${ }^{27}$ selecionaram 28 pessoas cujos relatos indicaram maior frequência de quedas nos últimos 12 meses e após os 65 anos de idade. Os resultados indicaram que a deterioração da visão, o uso simultâneo de medicamentos (especialmente diuréticos e psicoativos) e a redução da flexibilidade (quadril e tornozelos) parecem associar-se com a frequência de quedas nos períodos observados. É importante que novas pesquisas sejam realizadas com esta população, porém de forma prospectiva. 
Quadro 3. Orientações e controle de segurança do idoso

\begin{tabular}{|c|c|}
\hline \multicolumn{2}{|l|}{ Checklist de segurança para casa } \\
\hline 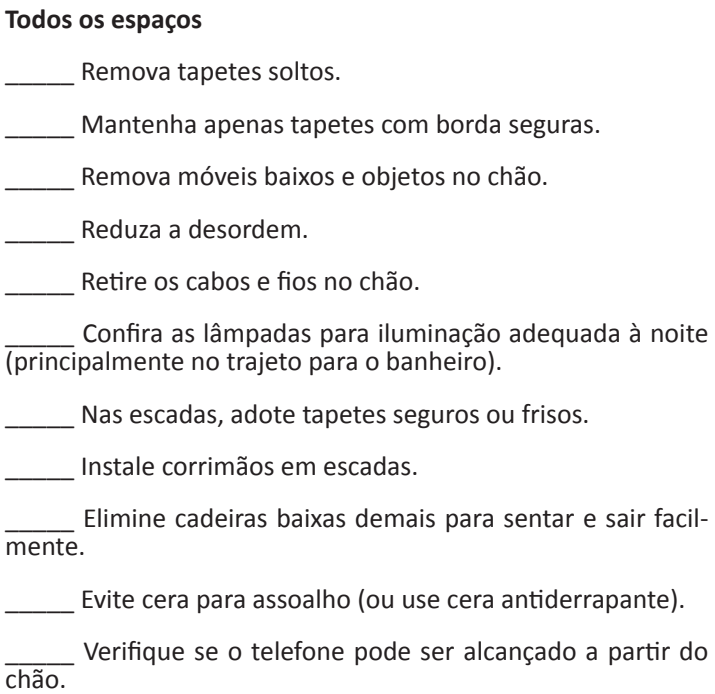 & 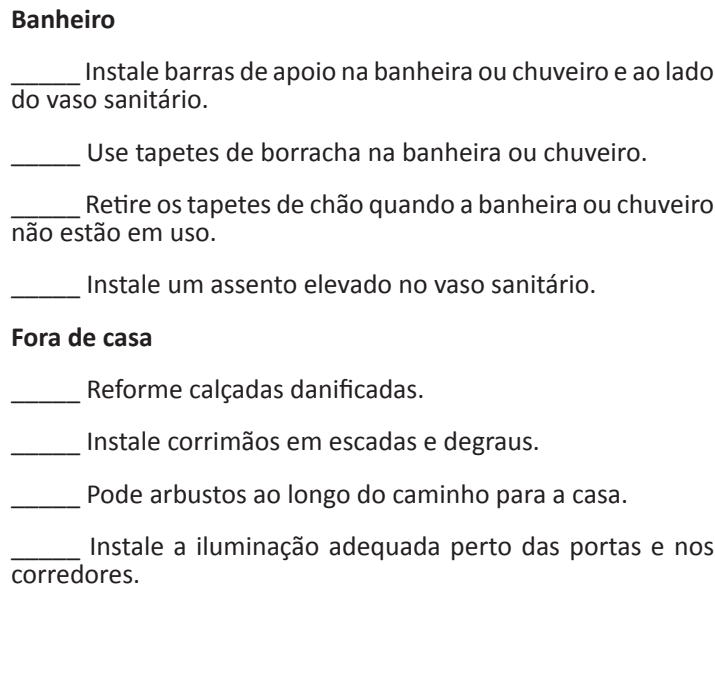 \\
\hline
\end{tabular}

Fonte: adaptado de Fuller ${ }^{20}$

\section{Artigos originais quanto às orientações e à abordagem fisioterapêutica na prevenção da queda em idosos}

Uma pesquisa realizada por Campbell e colaboradore ${ }^{28}$ teve por objetivo avaliar a eficácia e o custo-benefício de um programa de segurança em casa (com terapeuta ocupacional) e um programa de exercícios em casa (com fisioterapeuta) na redução de quedas e de injúrias oriundas destas em idosos com deficiência visual. Para tal, foram analisados 371 idosos, com idade igual ou superior a 75 anos, divididos randomicamente em quatro grupos: (a) programa de segurança em casa $(\mathrm{n}=$ 100); (b) programa de exercícios realizados em casa, com suplementação de vitamina $D(n=97)$; (c) intervenção do grupo (a) e (b) $(n=98)$ e (d) visitas sociais $(n=96)$. Nos resultados observou-se que o grupo (a) obteve melhor eficácia e custo-benefício em relação aos demais grupos e, ainda, que a intervenção realizada pelo grupo (b) não influenciou na redução de quedas e dos comprometimentos secundários a elas.

No trabalho de Barnett e colaboradores ${ }^{29}$ verificou-se melhora significativa no equilíbrio, força, resistência muscular, agilidade e diminui- ção no risco de quedas em gerontes. A intervenção constou de um programa de exercícios de resistência muscular, flexibilidade, equilíbrio e coordenação motora com orientações para a realização de exercícios em domicílio. O quadro 3 também traz estas orientações, adaptadas do trabalho de Fuller. ${ }^{20}$

Embora o enfoque da presente pesquisa não tenha se restringido necessariamente aos idosos com osteoporose, um estudo randomizado e controlado com essa amostra $(\mathrm{N}=96$, idade $=$ 71,0 \pm 4,7 anos; 90 mulheres) foi realizado por Smulders e colaboradores em um hospital e teve por objetivo avaliar a eficácia do Programa de Prevenção de Quedas Nijmegen (NFPP) para idosos com osteoporose e história de queda. ${ }^{30}$ Após avaliação inicial, os participantes foram aleatoriamente designados para o exercício - NFPP (GE; $\mathrm{n}=50$; por cinco semanas e três dias) ou grupo controle (GC; $\mathrm{n}=46$; cuidados habituais). A variável de desfecho primária foi a taxa de queda, medida por meio de calendários de queda mensal durante um ano. Os desfechos secundários foram o equilíbrio (Activity-specific Balance Confidence Scale), a qualidade de vida (QV; Quality of Life Questionnaire of the European Foundation for Osteoporosis), e nível de atividade física (LASA Physical Activity Questionnaire, hodômetro), ava- 
liados após o tratamento, posterior ao programa e após um ano de follow-up. Em seus resultados, a taxa de queda no grupo de estudo foi inferior à do grupo de controle (0,72 vs 1,18 quedas / pessoa -ano, razão de risco de 0,61 , intervalo de confiança de $95 \%$ de $0,40-0,94)$. O equilíbrio no grupo de estudo aumentou 13,9\% ( $p=0,001)$. Não houve diferenças em ambos os grupos tanto na qualidade de vida quanto nos níveis de atividade física. Desta forma, o estudo inferiu que o NFPP para idosos com osteoporose foi eficaz em diminuir o número de quedas e em melhorar o equilíbrio, dando sustentação à importância de um programa de exercícios físicos na redução de quedas. Este interfere nas perdas fisiológicas decorrentes do envelhecimento, minimizando-as.

A plataforma vibratória é um tipo de tratamento recente na fisioterapia, e seu uso tem aumentado nos últimos anos para os idosos. Embora a exposição à vibração seja considerada por alguns como perigosa, uma pesquisa recente centrou-se em benefícios potenciais nos quais os princípios físicos de vibrações forçadas são discutidos em relação à vibração como uma modalidade de exercício. As respostas fisiológicas agudas encontradas variam desde o tendão do músculo isolado até a vibração do corpo inteiro, assim como os efeitos do treinamento sobre a musculatura, densidade mineral óssea e postura. ${ }^{31}$ Evidências sugerem que o exercício de vibração parece provocar um efeito de aquecimento específico, e que o seu treinamento parece melhorar a força muscular, embora os benefícios potenciais em comparação às formas tradicionais de exercício resistido ainda não estejam claros. O treino na plataforma vibratória também parece melhorar a estabilidade de marcha e reduzir o risco de queda, especialmente em idosos frágeis (institucionalizados ou hospitalizados). Todavia, é importante a orientação feita por profissional qualificado, pois o uso desajustado da vibração de corpo inteiro pode levar a problemas de saúde, especialmente em tecidos osteoarticulares. ${ }^{32}$

Outra forma moderna e diferenciada de tratamento é o treinamento em realidade virtual, uma tecnologia emergente que pode ajudar a promover a atividade física e combinar os pontos fortes do exercício interior (indoor) e exterior (outdoor), que tem sido recentemente proposta pelo potencial de incentivar a prática do exercício físico em gerontes. Para os adultos mais velhos, os ambientes interativos (virtuais) podem influenciar o controle postural e de quedas, estimulando os sinais sensoriais que são responsáveis por manter o equilíbrio e a orientação. ${ }^{33}$

Outros poucos trabalhos também foram realizados com intervenção. ${ }^{34-37}$ Entretanto, a publicação sobre intervenções na população brasileira (prevenção da queda em idoso) ainda é escassa.

\section{Conclusões}

O trabalho com gerontes confronta o fisioterapeuta com uma série de desafios. Os casos em que a queda está associada variam muito: afecções osteomusculares, neurológicas e cardiovasculares que podem estar representadas em um grupo de pessoas ou até num mesmo paciente. A combinação e a interligação de problemas médicos, psicológicos, reabilitativos e socioeconômicos requerem a atenção do fisioterapeuta. As diferenças na manifestação de cada doença e o envelhecimento que se processa em cada indivíduo mostram a complexidade do desafio, tornando esta área ainda mais instigante para a profissão de fisioterapia.

No entanto, também foi observado que, em decorrência do processo natural da senescência, idosos aparentemente saudáveis também estão propensos ao risco de quedas, principalmente na vigência do sedentarismo. Para este grupo, também foi observado que um programa de exercícios físicos e orientações aperfeiçoa a independência física e melhora a qualidade de vida, uma vez que minimiza a ocorrência de quedas e as complicações decorrentes da mesma. Outros assuntos levantados a partir de terapias recentes foram os treinamentos em plataforma vibratória e em realidade virtual. Todavia, ainda são poucos os trabalhos produzidos sobre esta população, principalmente no Brasil, abrindo-se um novo caminho de descobertas de um treinamento diferenciado.

\section{Referências}

1. National Ageing and Research Institute. An analysis of research on preventing falls and falls injury in older people: community, residential and hospital settings. Canberra: Australian Government Department of Health and Ageing; 2004. 
2. Gonçalves LG, Vieira ST, Siqueira FV, Hallal PC. Prevalência de quedas em idosos asilados do município de Rio Grande, RS. Rev Saúde Pública. 2008;42(5):936-45.

3. Oliver D, Daly F, Martin F, McMurdo ME. Risk factors and risk assessment tools for falls in hospital in-patients: a systematic review. Age Ageing. 2004;31(2):121-8.

4. American Geriatrics Society, British Geriatrics Society, and American Academy of Orthopaedic Surgeons Panel on Falls Prevention. Guideline for the Prevention of Falls in Older Persons. J Am Geriatr Soc. 2001;49(5):664-72.

5. Siqueira FV, Facchini LA, Piccini RX, Tomasi E, Thumé E, Silveira DS, et al. Prevalência de quedas em idosos e fatores associados. Rev Saúde Pública. 2007;41(5):749-56.

6. Cesari M, Landi F, Torre S, Onder G, Lattanzio F, Bernabei R. Prevalence and risk factors for falls in an older community-dwelling population. J Gerontol A Biol Sci Med Sci. 2002;57(11):M721-5.

7. Fabrício SCC, Rodrigues RAP, Costa Junior ML. Causas e consequências de quedas de idosos atendidos em hospital público. Rev Saúde Pública. 2004;36(1):93-9.

8. Bhatt T, Espy D, Yang F, Pai YC. Dynamic gait stability, clinical correlates, and prognosis of falls among community-dwelling older adults. Arch Phys Med Rehabil. 2011;92(5):799-805.

9. Hofheinz M, Schusterschitz C. Dual task interference in estimating the risk of falls and measuring change: a comparative, psychometric study of four measurements. Clin Rehabil. 2010;2303(9):829-42.

10. Gonçalves DFF, Ricci NA, Coimbra AMV. Equilíbrio funcional de idosos da comunidade: comparação em relação ao histórico de quedas. Rev Bras Fisioter. 2009;13(4):216-23.

11. Donoghue D, Physiotherapy Research and Older People (PROP) group, Stokes EK. How much change is true change? The minimum detectable change of the Berg Balance Scale in elderly people. J Rehabil Med. 2009;41(5):32323-6.

12. Southard V, Dave M, Davis MG, Blanco J, Hofferber A. The multiple tasks test as a predictor of falls in older adults. Gait Posture. 2005;21(4):331-5.

13. Leal SMO, Borges EGS, Fonseca MA, Alves Junior ED, Cader S, Dantas EHM. Efeitos do treinamento funcional na autonomia funcional, equilíbrio e qualidade de vida de idosas. R Bras Ci Mov. 2009;17(3):61-9.

14. Alves ED Jr, Paula FL. The prevention of falls under the aspect of health promotion. Fit Perf J. 2008;7(2):123-9.

15. Oliver D, Martin F, Seed P. Preventing patient falls. Age Ageing. 2002;29(1):75-6.
16. Kron M, Loy S, Sturm E, Nikolaus T, Becker C. Risk indicators for falls in institutionalized frail elderly. Am J Epidemiol. 2003;158(7):645-53.

17. Tinetti ME, Kumar C. The patient who falls: "It's always a trade-off". JAMA. 2010;303(3):258-66.

18. Day L, Finch CF, Hill KD, Haines TP, Clemson L, Thomas M, et al. A protocol for evidence-based targeting and evaluation of statewide strategies for preventing falls among community-dwelling older people in Victoria, Australia. Inj Prev. 2011;17(2):e3.

19. Panel of Prevention of Falls in Older Persons, American Geriatrics Society and British Geriatrics Society. Summary of the Updated American Geriatrics Society/British Geriatrics Society clinical practice guideline for prevention of falls in older persons. J Am Geriatr Soc. 2011;59(1):148-57.

20. Fuller GF. Falls in the elderly. Am Fam Physician. 2000;61(7):459-68,473-4.

21. Hitcho EB, Krauss MJ, Birge S, Dunagan WC, Fischer I, Johnson S, et al. Characteristics and circumstances of falls in a hospital setting: a prospective analysis. J Gen Intern Med. 2004;19(7):732-9.

22. Perracini MR, Ramos LR. Fatores associados a quedas em uma coorte de idosos residentes na comunidade. Rev Saúde Pública. 2002;34(6):709-16.

23. Laessoe U, Hoeck HC, Simonsen O, Sinkjaer T, Voigt $M$. Fall risk in an active elderly population - can it be assessed? J Negat Results Biomed. 2007;6:2.

24. Stalenhoef PA, Diederiks JP, Knottnerus JA, Witte LP, Crebolder HF. The construction of a patient record-based risk model for recurrent falls among elderly people living in the community. Fam Pract. 2000;17(6):490-6.

25. Thrane G, Joakimsen RM, Thornquist E. The association between timed up and go test and history of falls: the Tromso study. BMC Geriatr. 2007;7:1.

26. Ziere G, Dieleman JP, Hofman A, Pols HA, Van der Cammen TJ, Stricker BH. Polypharmacy and falls in the middle age and elderly population. $\mathrm{Br}$ J Clin Pharmacol. 2006;61(2):218-23.

27. Guimarães JMN, Farinatti PTV. Análise descritiva de variáveis teoricamente associadas ao risco de quedas em mulheres idosas. Rev Bras Med Esporte. 2005;11(5):279-85.

28. Campbell AJ, Robertson MC, La Grow SJ, Kerse NM, Sanderson GF, Jacobs RJ, et al. Randomised controlled trial of prevention of falls in people aged $>$ or $=75$ with severe visual impairment: the VIP trial. BMJ. 2005;331(7520):817-20.

29. Barnett A, Smith B, Lord SR, Williams M, Baumand A. Community-based group exercise improves balance and reduces falls in at-risk older people: 
a randomised controlled trial. Age Ageing. 2003;32(4):407-14.

30. Smulders E, Weerdesteyn V, Groen BE, Duysens J, Eijsbouts A, Laan R, et al. Efficacy of a short multidisciplinary falls prevention program for elderly persons with osteoporosis and a fall history: a randomized controlled trial. Arch Phys Med Rehabil. 2010;91(11):1705-11

31. Rittweger J. Vibration as an exercise modality: how it may work, and what its potential might be. Eur J Appl Physiol. 2010;108(5):877-904.

32. Raschilas F, Blain H. [What can we think about whole-body-vibration in elderly people?]. Presse Med. 2010;39(10):1032-7.

33. de Bruin ED, Schoene D, Pichierri G, Smith ST. Use of virtual reality technique for the training of motor control in the elderly. Some theoretical considerations. Z Gerontol Geriatr. 2010;43(4):229-34.

Recebido: 08/03/2013.

Revisado: 26/10/2013.

Aprovado: 15/12/2013.
34. Miller KL, Magel JR, Hayes JG. The effects of a homebased exercise program on balance confidence, balance performance, and gait in debilitated, ambulatory community-dwelling older adults: a pilot study. J Geriatr Phys Ther. 2010;31(2):85-91.

35. Krampe J, Rantz MJ, Dowell L, Schamp R, Skubic $\mathrm{M}$, Abbott C. Dance-based therapy in a program of all-inclusive care for the elderly: an integrative approach to decrease fall risk. Nurs Adm Q. 2010;34(2):156-61.

36. Desai A, Goodman V, Kapadia N, Shay BL, Szturm T. Relationship between dynamic balance measures and functional performance in community-dwelling elderly people. Phys Ther. 2010;90(5):748-60.

37. Bauer C, Rietsch C, Groger I, Gassmann KG. [Mobility and safety for elderly (MoSi), a new intervention to improve mobility and gait in elderly people]. Z Gerontol Geriatr. 2009;42(5):340-4

\section{Samária Ali Cader}

Serviço de Fisioterapia. Policlínica Piquet Carneiro. Laboratório de Biociência da Motricidade Humana. Universidade do Estado do Rio de Janeiro. Rio de Janeiro, RJ, Brasil.

\section{Jorge da Silva Barboza}

Serviço de Fisioterapia. Policlínica Piquet Carneiro. Universidade do Estado do Rio de Janeiro. Rio de Janeiro, RJ, Brasil.

\section{Adalgisa leda Maiworn Bromerchenkel}

Serviço de Fisioterapia. Policlínica Piquet Carneiro. Universidade do Estado do Rio de Janeiro. Rio de Janeiro, RJ, Brasil. 